\title{
ON INVERSE LIMITS OF HOMOTOPY SETS
}

\author{
PETER J. KAHN ${ }^{1}$
}

ABSTRACT. An elementary proof is given that, under certain conditions on a space $F$, the homotopy set $[X, F]$ maps bijectively onto the inverse limit of homotopy sets determined by the finite subcomplexes of $X$. The only other satisfactory proof known requires the Brown representability theorem.

Throughout this note we deal only with based maps and based CW complexes. $X$ and $F$ will be such $C W$ complexes, and $\left\{X_{a}\right\}$ will be the set of finite subcomplexes of $X$, directed by inclusion. We assume that $F$ is connected $^{2}$ and that each homotopy group of $F$ is finite.

Theorem 1. The natural map

$$
[X, F] \stackrel{\pi_{X}^{0}}{\longrightarrow} \underset{a}{\lim }\left[X_{\alpha}, F\right]
$$

is bijective.

This result is trivial when $X$ has dimension 0 or is a finite complex. Moreover, when $X$ is an increasing union of a sequence $\left\{X_{n}\right\}$ of subcomplexes such that $\pi_{X}^{0}$ is surjective for each $n$, then an inductive application of the homotopy extension property yields that $\pi_{X}^{0}$ is surjective. In particular, this gives surjectivity when $X$ is a countable CW complex. The problem is that no such straightforward argument seems to work for uncountable $X$.

Define $L_{F}^{0} X$ to be the inverse limit set in Theorem 1. The theorem may then be interpreted as saying that $L_{F}^{0}$ is a representable functor. This suggests a connection between Theorem 1 and the Brown representability theorem, and, indeed, Brown's theorem has been used to prove Theo-

Received by the editors September 6, 1973 and, in revised form, November 17, 1973.

AMS (MOS) subject classifications (1970). Primary 55E05; Secondary 55B20.

Key words and phrases. Inverse limit, homotopy set.

1 Partly supported by NSF Grant GP 33960X.

2 There need be no restriction on the number of components of $F$; our assumption is merely for convenience. 
rem $1[3],[5, \mathrm{p} .3 .18]$. The purpose of this note is to present a direct, elementary proof.

Theorem 1 is generally false without some kind of finiteness condition on $\pi_{i} F$ (e.g., see [4]), but the surjectivity of $\pi_{X}^{0}$, for all $X$, can be proved using an algebraic condition on $F[1],[2]$. The precise conditions for surjectivity are not yet well understood.

Now define

$$
L_{F}^{1} X=\underset{a}{\lim }\left[X X_{a} \times I \cup X \times \partial I, F\right],
$$

where $I$ is the unit interval. ( $L_{F}^{n}$ can be defined analogously for $n=$ $2,3, \cdots$, but they will not be needed.) We then have the following "MayerVietoris" sequence of based sets

$$
[X \times I, F] \stackrel{\pi_{X}^{1}}{\longrightarrow} L_{F}^{1} X \stackrel{i_{1}}{\underset{i_{0}}{\longrightarrow}}[X, F] \stackrel{\pi_{X}^{0}}{\longrightarrow} L_{F}^{0} X,
$$

in which $\pi_{X}^{1}$ is induced by restriction, as in $\pi_{X}^{0}$, and $i_{0}$ and $i_{1}$ are induced by the two natural inclusions of $X$ into $X_{a} \times I \cup X \times \partial I$. Since $i_{0}{ }^{\circ} \pi_{X}^{1}=$ ${ }_{1} \circ \pi_{X}^{1}$ is a bijection, $\pi_{X}^{1}$ is injective.

Lemma 1. The above sequence is exact at $[X, F]$.

By this we mean that $\pi_{X}^{0} \circ i_{1}=\pi_{X}^{0} \circ i_{0}$, which we take as obvious, and that if $\pi_{X}^{0}(a)=\pi_{X}^{0}(b)$, then $a=i_{0}(c), b=i_{1}(c)$ for some $c \in L_{F}^{1} X$.

Theorem 2. $\pi_{X}^{0}$ and $\pi_{X}^{1}$ are surjective.

Therefore, $\pi_{X}^{1}$ is bijective. Moreover, since $\pi_{X}^{1}$ is surjective, it follows that $i_{0}=i_{1}$, which, by Lemma 1 , forces $\pi_{X}^{0}$ to be injective. Thus, Theorem 2 implies Theorem 1.

By the remarks made earlier, we can obtain Theorem 2 for $X$ provided that we can prove it for every skeleton of $X$. It clearly holds for the 0 skeleton of $X$. Thus, we need only

Lemma 2. Let $Y$ be an $n$-dimensional $C W$ complex, $n \geq 1$, and let $X$ be a subcomplex of $Y$ containing the $(n-1)$-skeleton of $Y$. If $\pi_{X}^{k}$ is surjective, $k=0,1$, then so is $\pi_{Y}^{k}$.

The proofs of Lemmas 1 and 2 are based on the following two elementary facts:

Fact A. An inverse limit of nonempty finite sets is nonempty.

Fact B. Let $Y$ be a CW complex, $X$ a subcomplex of $Y$, and $F$ as before. Let $g: X \rightarrow F$ be any map. If $Y \backslash X$ consists of finitely many 
cells, then there are only finitely many homotopy classes rel $X$ of extensions $Y \rightarrow F$ of $g$.

Let $[Y, F]_{g}$ denote this set of homotopy classes. Its finiteness is a result of elementary obstruction theory. Fact $B$ applies when $X$ is empty, and it still holds, of course, if "rel $X$ " is deleted. Fact A is a direct consequence of König's lemma on finitely branching trees.

Proof of Lemma 1. For each $\alpha$, there are maps $j_{0}, j_{1}:\left[X_{a} \times I \cup X \times\right.$ $\partial I, F] \rightarrow[X, F]$ determined, as before, by the two natural inclusions of $X$. Since $\pi_{X}^{0}(a)=\pi_{X}^{0}(b)$, there exists, for each $a$, a $c_{a}$ satisfying $j_{0}\left(c_{a}\right)=$ $a, j_{1}\left(c_{a}\right)=b$. Letting $J_{a}$ be the set of all such $c_{a}$, we note that the $J_{a}$ 's, together with restriction maps, form an inverse system. Since $X_{a} \times I$ is a finite CW complex, Fact B implies that $J_{a}$ is finite. Fact $A$ then produces a $c \in \lim _{a} \subset L_{F}^{1} X$ with the required properties.

Proof of Lemma 2. The proof for $\pi_{X}^{1}$ is the same as that for $\pi_{X}^{0}$, and so for notational simplicity we do only the latter.

Case 1. $Y \backslash X$ has only finitely many cells.

We redefine $L_{F}^{0} X$ and $L_{F}^{0} Y$ by passing to cofinal subsets of $\left\{X_{a}\right\}$ and $\left\{Y_{a}\right\}$ : Namely, we use only $X_{a}$ containing the boundaries of all cells in $Y \backslash X$, and we use only $Y_{a}$ of the form $X_{\alpha} \cup(Y \backslash X)$. This enables us to define the restriction $L_{F}^{0} Y \rightarrow L_{F}^{0} X$.

Fix $\alpha$, and note that the commutative diagram

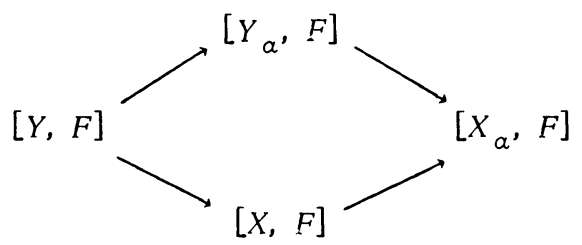

has, by homotopy extension, the following exactness property: If $\phi_{a} \epsilon$ $\left[Y_{a}, F\right]$ and $\psi \in[X, F]$ restrict to $\psi_{\alpha} \in\left[X_{\alpha}, F\right]$, then there exists a $\phi \in[Y, F]$ restricting to both $\phi_{a}$ and $\psi$. Let $J_{a}$ be the set of all such $\phi$. By Fact $B, J_{a}$ is finite.

If $\left\{\phi_{a}\right\} \in L_{F}^{0} Y$ is arbitrary, $\left\{\psi_{a}\right\} \in L_{F}^{0} X$ obtained from it by restriction, and if $\psi \in[X, F]$ satisfies $\pi_{X}^{0}(\psi)=\left\{\psi_{a}\right\}$, which $\psi$ exists by hypothesis, then the collection of all $J_{a}$ that we obtain as above for these $\phi_{a}, \psi_{a}$ and $\psi$ form a system directed by inclusion. By Fact $A, \cap J_{a}$ is nonempty. Each member $\phi$ satisfies $\pi_{Y}^{0}(\phi)=\left\{\phi_{a}\right\}$.

Case 2. The general case. So far we have not used the full strength of Fact B: Namely, that it applies to homotopy classes rel $X$. We now use this. 
We begin as before by choosing $\left\{\phi_{a}\right\} \in L_{F}^{0} Y$, restricting to $\left\{\psi_{a}\right\} \in L_{F}^{0} X$, and pulling back to a class $\psi \in[X, F]$ represented by some $g: X \rightarrow F$. Let $g$ index the cells of $Y \backslash X$, and let $\{\sigma\}$ be the collection of its finite subsets, directed by inclusion. Define $Y(\sigma)=X \cup\left\{e_{i} \mid i \in \sigma\right\}$, and let $p_{\sigma}:[Y(\sigma), F]_{g} \rightarrow[Y(\sigma), F]$ be the standard projection. In Case 1 , we showed that image $p_{\sigma}$ contains a nonempty finite set $J_{\sigma}$ such that $\pi_{Y(\sigma)}^{0}\left(J_{\sigma}\right)=\left\{\phi_{a}\right\} \mid Y(\sigma)$. Let $K_{\sigma}=p_{\sigma}^{-1}\left(J_{\sigma}\right)$ $C[Y(\sigma), F]_{g^{*}}$ Using $\mathrm{Fact} A$ and arguing as before, we conclude that there is a class $\left\{\phi_{\sigma}^{\prime}\right\} \in \lim K_{\sigma}$. For each singleton $\sigma$, let $f_{\sigma}^{\prime}$ represent $\phi_{\sigma}^{\prime}$, let $\phi^{\prime} \in[Y, F]_{g}$ be given by $\bigcup f_{\sigma}^{\prime}$, and let $\phi \in[Y, F]$ be given by the same map. For any $\sigma, \phi^{\prime} \mid[Y(\sigma), F]_{g}=\phi_{\sigma}^{\prime}$. Letting $Y_{a}$ be any finite subcomplex of $Y$, we choose $\sigma$ so that $Y(\sigma) \supset Y_{\alpha}$, and we obtain the desired conclusion that

$$
\pi_{Y}^{0}(\phi)=\left\{\phi \mid Y_{\alpha}\right\}=\left\{p_{\sigma}\left(\phi_{\sigma}^{\prime}\right) \mid Y_{\alpha}\right\}=\left\{\phi_{\alpha}\right\}
$$

\section{REFERENCES}

1. J. F. Adams, A variant of E. H. Brown's representability theorem, Topology 10 (1971), 185-198. MR 44 \#1018.

2. A. Deleanu, Remark on the Brown-Adams representability theorem, Syracuse University, 1971 (mimeo).

3. P. J. Kahn, Brown's repres entability theorem for compact functors, Cornell University, Ithaca, N. Y., 1972 (mimeo).

4. J. Milnor, On axiomatic homology theory, Pacific J. Math. 12 (1962), 337341. MR 28 \#2544.

5. D. Sullivan, Geometric topology. Part I (revised April, 1971), M.I.T. photo offset. 14850

DEPARTMENT OF MATHEM ATICS, CORNELL UNIVERSITY, ITHACA, NEW YORK

Current address: Mathematisches Institüt der Universität Heidelberg, 6900 Heidelberg 1 im Neuenheimer Feld 288, Heidelberg, West Germany 